\title{
DIAGNÓSTICO DA RELAÇÃO ENTRE A ARBORIZAÇÃO E A DIVERSIDADE DE ABELHAS SEM FERRÃO (APIDAE: MELIPONINI) NO CAMPUS TAPAJÓS E NO BOSQUE MEKDECE LOCALIZADOS EM SANTARÉM, PA
}

\author{
Nayara dos Santos Mesquita'; Graciene Conceição dos Santos²; Nayane dos Santos Mesquita3; Rayane dos \\ Santos Mesquita ${ }^{4}$; Franciléia dos Santos Mesquita ${ }^{5}$; Rafael Rode ${ }^{6}$; Rogério Souza Ribeiro ${ }^{7}$; Alanna do Socorro \\ Lima da Silva ${ }^{8}$.

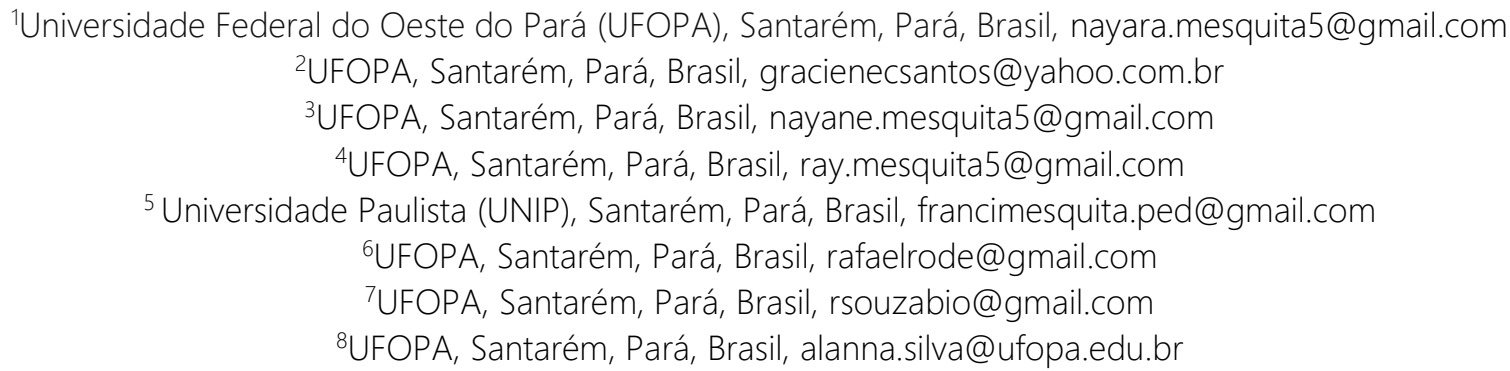

RESUMO: O estudo objetivou caracterizar a significância da arborização como instrumento de conservação da biodiversidade de abelhas sem ferrão no Campus Tapajós e no Bosque Mekdece, localizados na cidade de Santarém, PA. Cada ninho encontrado foi georreferenciado, fotografado, mediu-se sua altura em relação ao solo, para substratos arbóreos mediu-se também a Circunferência do tronco a 1,30 m. As abelhas coletadas foram identificadas a partir da arquitetura de entrada do ninho e de chave de identificação. Verificou-se a riqueza, abundância e diversidade. Foram encontrados ao todo 12 ninhos, pertencentes a 6 espécies diferentes: Trigona guianae $(\mathrm{N}=2)$, Melipona (Melikerria) interrupta $(\mathrm{N}=3)$, Melipona seminigra aff pernigra $(\mathrm{N}=1)$, Trigona pallens $(\mathrm{N}=3)$, Tetragona clavipes $(\mathrm{N}=2)$, Scapitotrigona sp. $(\mathrm{N}=1)$. Os índices de diversidade Shannon-Wiener $\left(\mathrm{H}^{\prime}\right)$ foram 1,56 no Campus Tapajós e 1,04 no Bosque Mekdece, já os índices de equitabilidade de Pielou (J) foram 0,97 no Campus Tapajós e 0,95 no Bosque Mekdece, a densidade dos ninhos foi de 1 ninho por hectare (Campus) e 3 ninhos por hectare (Bosque). Com relação à nidificação em árvores, no Campus Tapajós foram identificados 5 ninhos em Dalbergia spruceana e 1 ninho em Copaifera martii, já no Bosque Mekdece identificou-se 3 ninhos em uma mesma espécie arbórea (Inga heterophylla). Quanto à nidificação de abelhas no solo foram encontrados 2 ninhos da espécie Trigona guianae no Campus e 1 ninho de Trigona pallens no Bosque. Constatou-se nos dois locais de estudo que a maioria dos ninhos (75\%) estava associada a substratos arbóreos e $25 \%$ dos ninhos encontrados estavam localizados no solo.

PALAVRAS-CHAVE: Abelhas sem ferrão, Conservação, Nidificação. 


\title{
DIAGNOSIS OF THE RELATIONSHIP BETWEEN THE WETLANDS AND THE DIVERSITY OF STINGLESS BEES (APIDAE: MELIPONINI) IN THE CAMPUS TAPAJÓS AND IN THE MEKDECE FOREST LOCATED IN SANTARÉM, PA
}

\begin{abstract}
The study aimed to characterize the significance of afforestation as an instrument to conserve the biodiversity of stingless bees in the Tapajós Campus and the Mekdece Forest, located in the city of Santarém, PA. Each nest was georeferenced, photographed, its height was measured in relation to the soil, for arboreal substrates the circumference of the trunk was also measured at $1.30 \mathrm{~m}$. The collected bees were identified from the nest entry architecture and identification key. We found wealth, abundance, and diversity. Twelve nests were found in 6 different species: Trigona guianae $(N=2)$, Melipona (Melikerria) interrupta $(N=3)$, Melipona seminigra aff pernigra $(N=1)$, Trigona pallens $(N=3)$, Tetragona clavipes $(N=2)$, Scapitotrigona sp $(N=1)$. The Shannon-Wiener $\left(H^{\prime}\right)$ diversity indexes were 1.56 in the Tapajós Campus and 1.04 in the Mekdece Forest, while the Pielou (J) equitability indexes were 0.97 in the Tapajós Campus and 0.95 in the Mekdece Forest, the nest density was 1 nest per hectare (Campus) and 3 nests per hectare (Forest). In relation to the nesting in trees, in the Tapajós Campus 5 nests were identified in Dalbergia spruceana and 1 nest in Copaifera martii. In the Mekdece Forest, 3 nests were identified in the same tree species (Inga heterophylla). Concerning the nesting of bees in the soil were found 2 nests of the species Trigona guianae in the Campus and 1 nest of Trigona pallens in the Forest. It was found in the two study sites that most of the nests (75\%) were associated with arboreal substrates and $25 \%$ of the nests found were located in the soil.
\end{abstract}

KEYWORDS: Conservation, Nesting, Stingless bees.

\section{DIAGNÓSTICO DE LA RELACIÓN ENTRE LA ARBORIZACIÓN Y LA DIVERSIDAD DE ABEJAS SIN AGUIJÓN (APIDAE: MELIPONINI) EN EL CAMPUS TAPAJÓS Y EN EL BOSQUE MEKDECE LOCALIZADOS EN SANTARÉM, PA}

RESUMEN: El estudio objetivó caracterizar la significancia de la arborización como instrumento de conservación de la biodiversidad de abejas sin aguijón en el Campus Tapajós y en el Bosque Mekdece, ubicados en la ciudad de Santarém, PA. Cada nido encontrado fue georreferenciado, fotografiado, midió su altura en relación al suelo, para sustratos arbóreos se midió también la Circunferencia del tronco a 1,30m. Las abejas recolectadas fueron identificadas a partir de la arquitectura de entrada del nido y de la clave de identificación. Se verificó la riqueza, abundancia y diversidad Se encontraron en total 12 nidos, pertenecientes a 6 especies diferentes: Trigona guianae $(\mathrm{N}=2)$, Melipona (Melikerria) interrupta $(\mathrm{N}=3)$, Melipona seminigra aff pernigra $(\mathrm{N}=1)$, Trigona pallens $(\mathrm{N}=3)$, Tetragona clavipes $(\mathrm{N}=2)$, Scapitotrigona sp. $(\mathrm{N}=1)$. Los índices de diversidad Shannon-Wiener ( $\mathrm{H}$ ') fueron 1,56 en el Campus Tapajós y 1,04 en el Bosque 
Mekdece, ya los índices de equidad de Pielou (J) fueron 0,97 en el Campus Tapajós y 0,95 en el Bosque Mekdece, la densidad de los nidos fue de 1 nido por hectárea (Campus) y 3 nidos por hectárea (Bosque). Con respecto a la nidificación en árboles, en el Campus Tapajós se identificaron 5 nidos en Dalbergia spruceana y 1 nido en Copaifera martii, ya en el Bosque Mekdece se identificaron 3 nidos en una misma especie arbórea (Inga heterophylla). En cuanto a la nidificación de abejas en el suelo se encontraron 2 nidos de la especie Trigona guianae en el Campus y 1 nido de Trigona pallens en el Bosque. Se constató en los dos lugares de estudio que la mayoría de los nidos (75\%) estaban asociados a sustratos arbóreos y el 25\% de los nidos encontrados estaban localizados en el suelo.

PALABRAS CLAVE: Abejas sin aguijón, Conservación, Nidificación.

\section{INTRODUÇÃO}

Pertencentes à subtribo Meliponina, os meliponídeos apresentam tamanho que varia de muito pequeno a médio, e estão agrupadas na família Apidae, assim como as demais abelhas sociais (SILVEIRA et al., 2002). As abelhas Meliponas popularmente conhecidas como abelhas sem ferrão ou abelhas indígenas, são insetos sociais de grande diversidade e de ampla distribuição geográfica, no Brasil, são encontradas mais de 300 espécies, distribuídas em 27 gêneros (KERR; FILHO 1999, SILVEIRA et al., 2002). Os ninhos dos meliponídeos apresentam arquitetura complexa, cada espécie tem uma estrutura peculiar na entrada do ninho, que geralmente a identifica. Essas abelhas utilizam diferentes tipos de substratos para nidificação, como ocos em árvores, cupinzeiros abandonados, raízes e até cipós (ROUBIK, 2006). A entrada do ninho é formada por um tubo de cera ou cerume, cujo comprimento varia com a espécie, sendo ausente em algumas (CAMARGO; PEDRO, 2008). Roubik (1989), estudando espécies da floresta, sugeriu que as abelhas são oportunistas em selecionar a localização do ninho e usam qualquer espécie de árvore que apresente cavidade de dimensões aproveitáveis.

As abelhas, também são consideradas bons indicadores biológicos, uma vez que realizam a polinização de diversas flores e utilizam plantas como substrato para construção de seus ninhos, podendo ser dessa maneira vulneráveis às alterações e fragmentação dos habitats (CAMARGO, 1970; MARTINS et al., 2004; CAMARGO; 
PEDRO, 2007; RÊGO, 2008) sendo que essa vulnerabilidade torna-se maior, quanto mais especializada uma espécie é em determinado recurso (MARTINS et al., 2013). A riqueza de espécies em uma área pode ser modificada por alterações no biótopo, que podem reduzir os locais disponíveis para nidificação (LINSLEY, 1958). A perda de habitats naturais tem efeitos bastante perturbadores sobre as comunidades dos meliponídeos já que a reprodução desse grupo se dá por enxameagem progressiva, que consiste num longo período de dependência entre a colônia-mãe e colônia-filha (NOGUEIRA-NETO, 1997). Dessa forma, a redução de áreas verdes pode ocasionar diminuição das populações locais.

Uma forma bastante eficiente de diagnosticar a situação atual das populações de abelhas sem ferrão é estudar a ecologia de nidificação destas abelhas, o que, de acordo com Teixeira (2003), possibilita extrair informações ecológicas importantes, como riqueza e diversidade das espécies residentes, espécies mais abundantes e as mais raras, densidade de ninhos e substratos utilizados, o que pode contribuir para a elaboração de planos de manejo e monitoramento de áreas naturais e urbanizadas. Sendo assim, este estudo objetivou caracterizar a significância da arborização como instrumento de conservação da biodiversidade de abelhas sem ferrão no Campus Tapajós e no Bosque Mekdece localizados na cidade de Santarém, PA.

\section{MATERIAL E MÉTODOS}

O estudo foi realizado na cidade de Santarém - PA $\left(02^{\circ} 26^{\prime} 35^{\prime \prime} \mathrm{S} 54^{\circ} 42^{\prime} 30^{\prime \prime} \mathrm{W}\right)$, que apresenta o clima dominante quente e úmido, característico das Florestas Tropicais, a temperatura média anual varia de $25^{\circ} \mathrm{C}$ a $28^{\circ} \mathrm{C}$. A precipitação pluvial média anual é de 1920 mm, com maior intensidade no período de dezembro a maio, e menor intensidade nos meses de junho a novembro correspondente ao período mais seco (INMET, 2017).

Os locais de estudo foram o Campus Tapajós da Universidade Federal do Oeste do Pará com uma área de 12 ha e coordenadas centrais $2^{\circ} 25^{\prime} \mathrm{S}$ e $54^{\circ} 44^{\prime} \mathrm{W}$, 
e o Bosque Mekdece, um fragmento florestal que apresenta uma área de 1,4 ha com as coordenadas centrais $2^{\circ} 25^{\prime} \mathrm{S}$ e $54^{\circ} 44^{\prime} \mathrm{W}$.

As observações ocorreram entre os meses de setembro a julho de 2017 entre 08h:00min e 12h:00min. Houve a exclusão dos meses mais frios do ano: dezembro, janeiro e março, por conta da diminuição da atividade externa das abelhas. As observações foram realizadas em dias de boas condições climáticas e dias ensolarados, sem ventos fortes ou em baixas temperaturas do inverno. Essas condições maximizam as atividades externas das abelhas e facilitam sua localização. De maneira geral, tanto no Campus Tapajós quanto no Bosque Mekdece todas as áreas foram vistoriadas à procura de ninhos de Meliponídeos, observando-se árvores vivas e mortas, paredes e edificações.

Para cada ninho encontrado, houve o registro fotográfico da entrada, a sua localização registrada por GPS, registrouse o tipo de substrato, o número de ninhos por substratos e foi aferida a altura do ninho em relação ao solo, quando se tratava de substratos arbóreos foi medida a Circunferência do tronco a 1,30m de altura (Circunferência à altura do peito(AP). Foram coletadas 5 abelhas em cada ninho com auxílio de um puçá e as espécies foram identificadas a partir da arquitetura da entrada e dos aspectos morfológicos da abelha a partir de chave de identificação (SILVEIRA et al., 2002) com auxílio de estéreo microscópios e os substratos arbóreos identificados pelo identificador botânico da instituição.

A análise dos dados foi através da densidade dos ninhos, abundância relativa, correlação de Pearson, o índice de diversidade Shannon-Wiener $\left(H^{\prime}\right)$ e complementado pelo índice de equibilidade de Pielou (J'). A densidade foi calculada a partir da fórmula: $d=$ número total dos ninhos/área total, o Índice de Shannon foi dado pela fórmula: $H^{\prime}=\Sigma$ pi.Inpi, em que $H$ é o componente de "riqueza" de espécies, e pi é a proporção de ninhos de cada espécie dada por fi/N, sendo que fi é o número de ninho de cada espécie e N o número total de ninhos na amostra, complementado por Pielou (J') , dado pela fórmula: $J^{\prime}=H^{\prime} / H^{\prime} \max$; em que 
$H^{\prime} \max .=$ InSe, sendo Se, o número total de ninhos da espécie de abelha considerada. Este índice (J') pode variar de 0 a 1. Quanto mais próximo de 0 for 0 índice, mais heterogênea será a distribuição das espécies; e quanto mais próximo de 1, mais homogênea. Os cálculos foram realizados com o auxílio do software Microsoft Excel, versão 2012.

\section{RESULTADOS E DISCUSSÃO}

Foram encontrados ao todo 12 ninhos, sendo 8 ninhos no Campus Tapajós e 4 ninhos no Bosque Mekdece, pertencentes a 6 espécies diferentes: Trigona guianae (Cockerell, 1910) (N=2), Melipona (Melikerria) interrupta (Latreille, 1811) $(\mathrm{N}=3)$, Melipona (Michmelia) seminigra aff. pernigra (Moure \& Kerr, 1950) $(\mathrm{N}=1)$, Trigona pallens (Fabricius, 1798) (N=3), Tetragona clavipes (Fabricius, 1804) (N=2), Scapitotrigona sp. $(\mathrm{N}=1)$. Os trabalhos de Vilhena (2009) e Souza (2005a) apresentaram 11 espécies de meliponídeos em contrapartida das 6 encontradas nos locais de estudo, o que indica que a diversidade é semelhante, pois as condições ambientais também se assemelham, ratificando o endemismo das abelhas indígenas sem ferrão na Amazônia. Já em Frazão (2009), a riqueza de meliponídeos foi alta, apresentando 35 espécies, sendo que o gênero mais abundante foi Trigona. As abundâncias relativas das espécies que ocorreram no presente estudo encontram-se nas Figuras 1 e 2 .

Ocorreram no presente estudo as seguintes espécies: Trigona guianae, com porte corpóreo médio, Tetragona clavipes e Trigona pallens, espécies de pequeno porte corpóreo, todas generalistas e adaptadas ao ambiente urbano. Em estudo feito por Oliveira et al. (1995), em experimentos de fragmentação florestal em Manaus, Trigona guianae foi encontrada tanto na mata contínua, como em fragmentos florestais de 1 ha, 10 ha e 100 ha (apenas não foi encontrada em área desmatada), todos na terra firme. Em estudo feito por Gonçalves et al. (1996), a espécie foi encontrada em ambiente de mata secundária fortemente antropizada. Na Colômbia foi encontrado ninho em altitude de 576m (VERGARA et al., 1986). Tetragona clavipes, em estudo feito pelos 
mesmos autores, somente foi encontrada em áreas desmatadas de terra firme, e em ambiente de mata secundária fortemente antropizada. Trigona pallens, também foi encontrada em áreas de mata secundária totalmente antropizadas, entretanto nos estudos realizados por Camargo e Roubik (1991), foi encontrado ninho em floresta densa de árvores emergentes.

Os substratos predominantemente observados nos dois locais de estudo encontram-se na Figura 3 e na Figura 4.

Figura 1. Abundância de ninhos de abelhas sem ferrão localizados no Campus Tapajós, Santarém, Pará, Brasil.

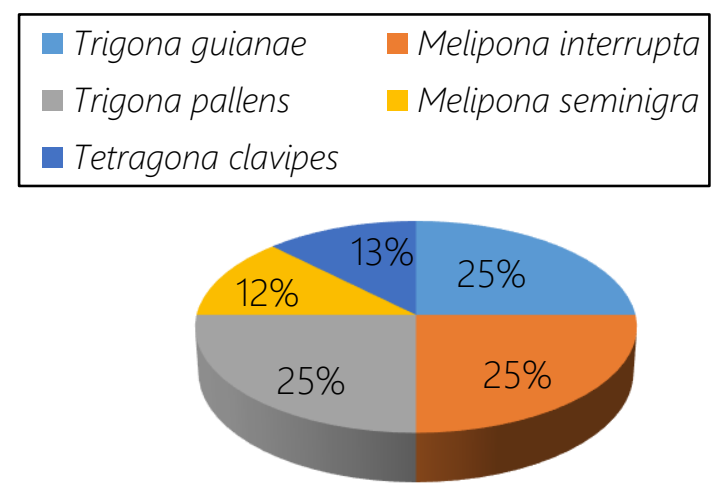

Figura 2. Abundância de ninhos de abelhas sem ferrão localizados no Bosque Mekdece, Santarém, Pará, Brasil.

- Melipona interrupta Trigona pallens

- Tetragona clavipes $\quad$ Scaptotrigona sp.

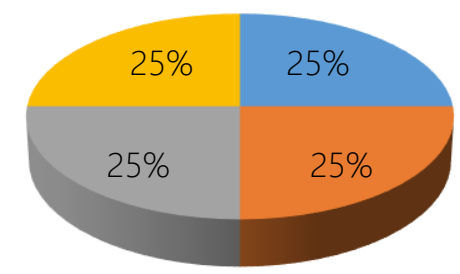


Figura 3. Distribuição dos ninhos em função do sítio de nidificação no Campus Tapajós, Santarém, Pará, Brasil.

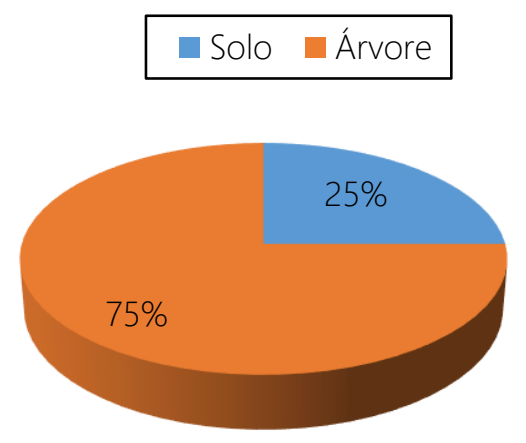

Figura 4. Distribuição dos ninhos em função do sítio de nidificação no Bosque Mekdece, Santarém, Pará, Brasil.

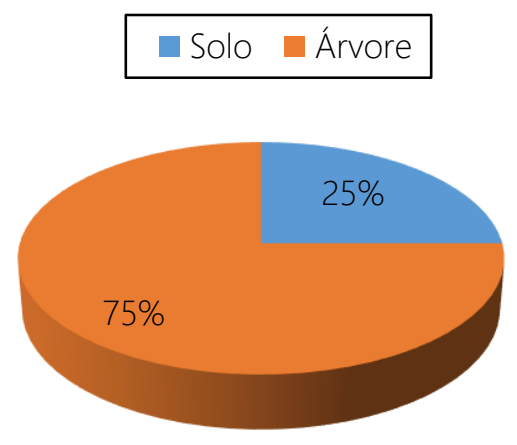

No Campus Tapajós foram Dalbergia spruceana (Jacarandá do Pará). encontrados dois casos de ninhos Todas as espécies nidificaram em associados, no primeiro caso houve Dalbergia spruceana, com exceção de associação de Melipona (Melikerria) Trigona pallens (Figura 6D) que também interrupta (Figura 5B) e Melipona nidificou em Copaifera martii (Michmelia) seminigra aff pernigra (Figura (Copaibarana- Caesalpiniaceae) e Trigona 5D), e no segundo caso entre Melipona guianae (Figuras 6A e 6B) ( $N=2$ ) que (Melikerria) interrupta (Figura 5A) e nidificou no solo. No Bosque Mekdece Tetragona clavipes (Figura 7C), ambos em houve associação de ninhos entre as 
espécies Scaptotrigona sp (Figura 7B), Tetragona clavipes (Figura 7D) e Melipona (Melikerria) interrupta (Figura 5C), em que todas nidificaram em Inga heterophylla (Ingá- Fabaceae). Os dados de nidificação segundo as espécies encontram-se na Tabela 1.

Com relação à nidificação em árvores, no Campus Tapajós foram identificados 6 ninhos pertencentes a duas espécies vegetais da família Fabaceae, em 4 árvores diferentes de Dalbergia spruceana (Jacarandá do Pará, N=5), e Copaifera martii (Copaibarana, $N=1$ ), no Bosque Mekdece identificou-se 3 ninhos $(\mathrm{N}=3)$ em Inga heterophylla (ingá). Martins et al.
(2004) observaram a utilização de 12 espécies arbóreas por meliponídeos na caatinga e $75 \%$ destes ninhos ocorreram em apenas duas espécies de árvores. Rêgo e Brito (1996) verificaram a utilização de apenas duas espécies vegetais para nidificação por meliponídeos em cerrado maranhense. Segundo os autores, Salvertia convallariodora (Vochysiaceae) foi a espécie vegetal que abrigou maior diversidade de espécies de abelhas sem ferrão e também o substrato de nidificação utilizado pela maioria dos meliponídeos (88\%; $n=22)$, sendo os demais ninhos encontrados em ocos de Platonia insignis- Clusiaceae.

Figura 5. Ninhos de meliponídeos encontrados nos locais de estudo. A. (S 2²5' 6.5" W 54

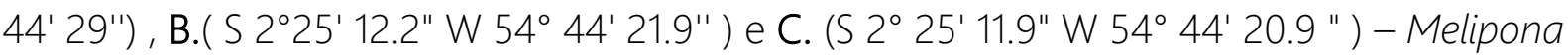
(Melikerria) interrupta (Latreille, 1811). D. (S 2²5' 12.2" W $54^{\circ} 44^{\prime} 21.9^{\prime \prime}$ )- Melipona (Michmellia) seminigra aff. pernigra (Moure \& Kerr, 1950).

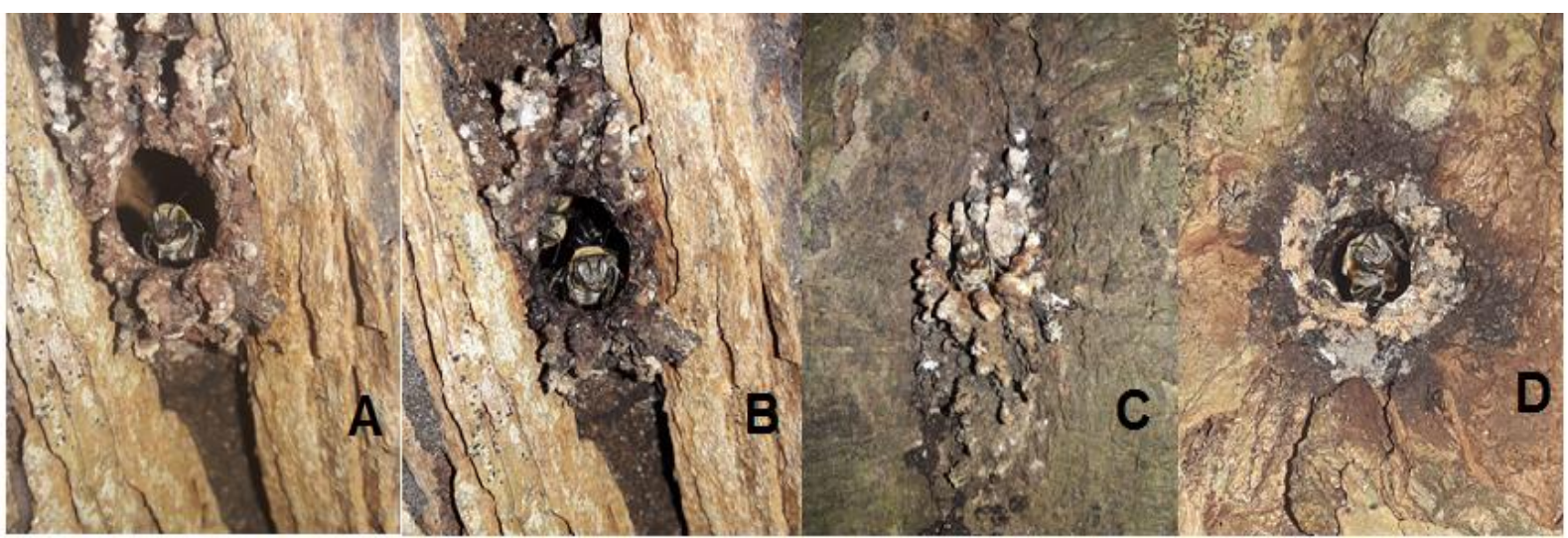


Figura 6. Ninhos de meliponídeos encontrados nos locais de estudo. A (S 2²5' 8.5"W $54^{\circ}$

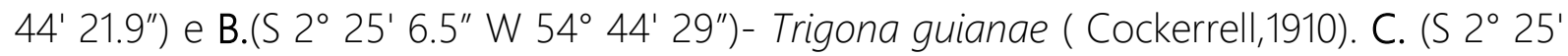
8.9" W 54 44' 25.1" ) e D. ( $2^{\circ} 25^{\prime} 12.2^{\prime \prime}$ W 54 44' 23.7") Trigona pallens (Fabricius, 1798).

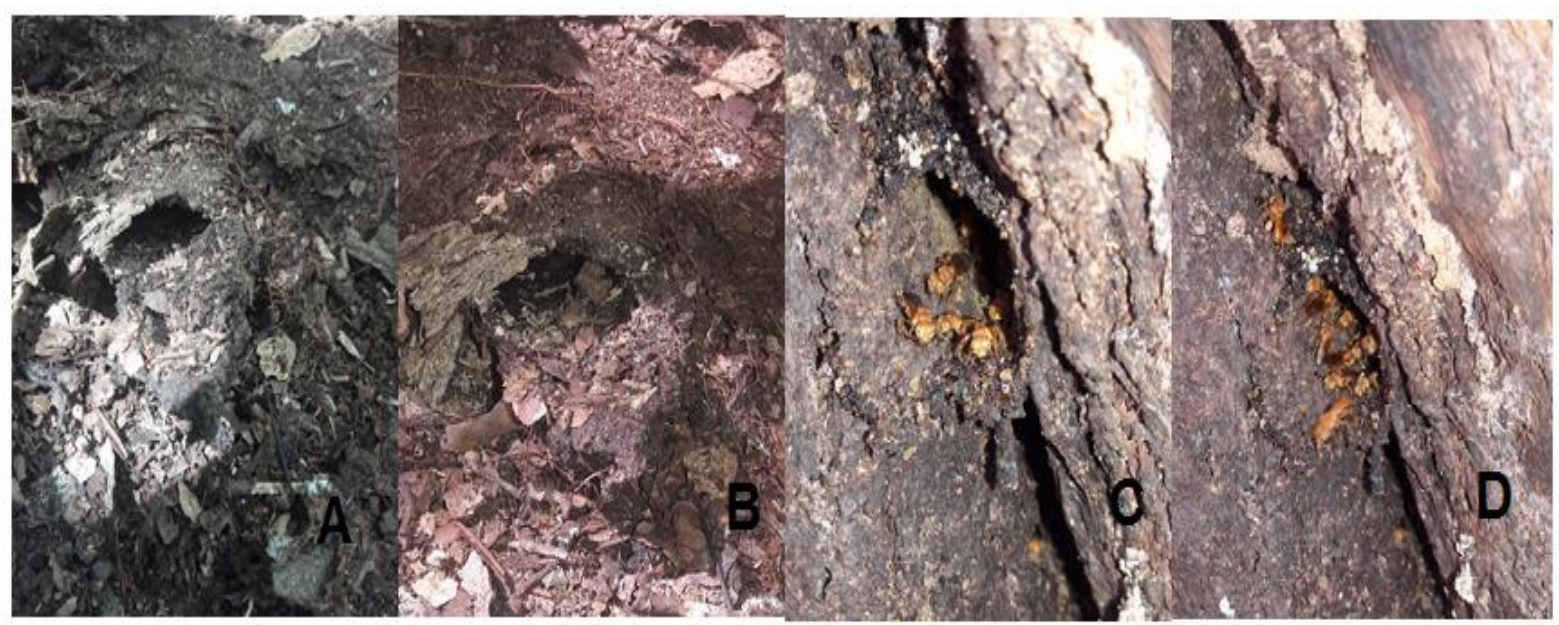

Figura 7. Ninhos de meliponídeos encontrados nos locais de estudo. A. (S $2^{\circ} 25^{\prime} 12.8^{\prime \prime} \mathrm{W}$ $\left.54^{\circ} 44^{\prime} 20^{\prime \prime}\right)$ - Trigona pallens (Fabricius, 1798). B. (S $2^{\circ} 25^{\prime} 11.9^{\prime \prime}$ W 54 44' 20.9") -

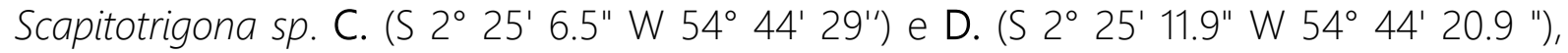
Tetragona clavipes (Fabricius, 1804).

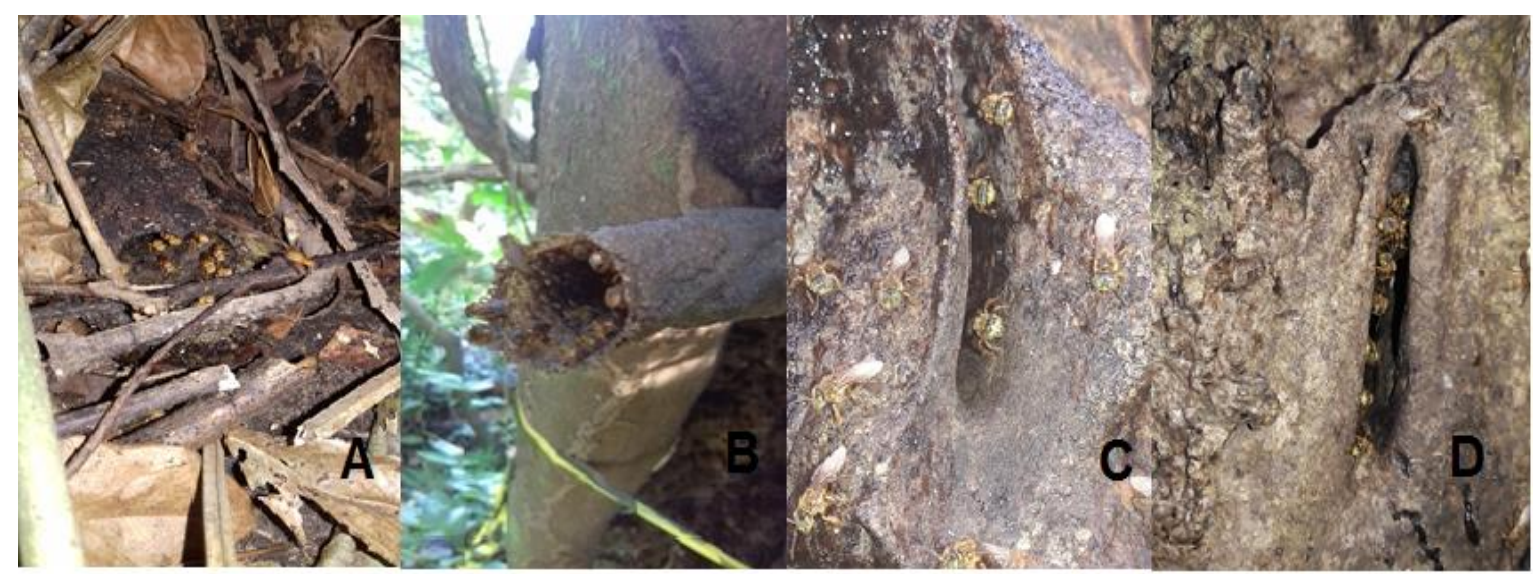

Diversos fatores podem estar ecológicos, como disponibilidade de influenciando a riqueza e a ocorrência de locais adequados para nidificação e oferta espécies de Meliponina em ambientes de recursos tróficos; fatores antropizados, destacando-se: fatores biogeográficos relacionados à própria 
distribuição geográfica de cada espécie e fatores filogenéticos inerentes a cada espécie de abelha sem ferrão (BATISTA, 2003; ELTZ et al., 2003; SLAA, 2006).

Melipona seminigra e Melipona interrupta foram encontradas no Campus Tapajós e Bosque Mekdece, ambientes com a presença de algumas árvores mais velhas e com maior diâmetro de tronco. Foram localizados ninhos em Dalbergia spruceana (Jacarandá do Pará- Fabaceae), árvore que possibilita volumosos ocos internos, assim como em Aquino et al. 2007, onde foram encontrados ninhos em árvores com diâmetro médio entre 36,21 cm e 41,68 cm, representando árvores mais velhas. Também foram encontrados ninhos em Inga heterophylla (IngáFabaceae) e Copaifera martii (Copaibarana- Caesalpiniaceae), árvore em que pode ser extraído o óleo de copaíba, corroborando o levantamento realizado por Oliveira et al. (2013) na Reserva de desenvolvimento Sustentável Amanã- RDSA e Reserva de desenvolvimento Sustentável MamirauáRDSM, em Manaus, onde foram encontrados ninhos destas espécies em ocos de árvores vivas conhecidas na região como matá-matá (Eschweilera albifl - Lecythidaceae), distantes em torno de 8 m do chão, orelha de cachorro (Crudia amazônica, Fabaceae), ambos na área da comunidade São João do Ipecaçu (setor Coraci- RDSA) e na embaúba (Cecropia sp., Urticaceae) na região da comunidade do Barroso (setor Barroso, RDSM). De acordo com Hubbell e Johnson (1977) e Roubik (1989), muitas espécies de abelhas sem ferrão são oportunistas na utilização de cavidades de árvores para nidificação. Este estudo mostra que abelhas sem ferrão nidificam em diferentes espécies arbóreas, entretanto, 75\% dos ninhos foram observados em apenas duas espécies de árvores.

- gênero Melipona, observado no estudo, é maior em locais que possuem a vegetação preservada, que provê recursos florais e locais de nidificação. Suas características são relacionadas ao maior porte corpóreo e hábito especialista, necessita de habitats com largas cavidades preexistentes em árvores, característico de árvores maiores e mais velhas que suportem acomodar 
essa maior estrutura de nidificação (ANTONINI et. al. 2013). Por sua biologia, é considerada um bioindicador da qualidade ambiental, sendo a riqueza de espécies de Meliponas inversamente relacionado com a distância da floresta e diretamente relacionado com a porcentagem de cobertura florestal (BROWN et al., 2001; ANTONINI et al., 2013).

Tabela 1. Dados da nidificação de abelhas sem ferrão localizados no Campus Tapajós e no Bosque Mekdece, Santarém, Pará, Brasil.

\begin{tabular}{lcccc}
\hline Espécie & Planta/local & Posicionamento & $\begin{array}{c}\text { Altura } \\
(\mathrm{m})\end{array}$ & CAP (cm) \\
\hline Trigona guianae & solo & $\mathrm{S} 2^{\circ} 25^{\prime} 8.5^{\prime \prime} 54^{\circ} 44^{\prime} 21.9^{\prime \prime} \mathrm{W}$ & solo & solo \\
Trigona guianae & solo & $\mathrm{S} 2^{\circ} 25^{\prime} 65^{\prime \prime} 54^{\circ} 44^{\prime} 29^{\prime \prime} \mathrm{W}$ & solo & solo \\
Melipona interrupta & Jacarandá do Pará & $\mathrm{S} 2^{\circ} 25^{\prime} 12.2^{\prime \prime} 54^{\circ} 44^{\prime} 21.9^{\prime \prime} \mathrm{W}$ & 0,95 & 174 \\
Melipona interupta & Jacarandá do Pará & $\mathrm{S} 2^{\circ} 25^{\prime} 65^{\prime \prime} 54^{\circ} 44^{\prime} 29^{\prime \prime} \mathrm{W}$ & 6,00 & 130 \\
Tetragona clavipes & Jacarandá do Pará & $\mathrm{S} 2^{\circ} 25^{\prime} 6.5^{\prime \prime} \mathrm{W} 54^{\circ} 44^{\prime} 29^{\prime \prime} \mathrm{W}$ & 6,00 & 130 \\
Trigona pallens & Jacarandá do Pará & $\mathrm{S} 2^{\circ} 25^{\prime} 12.2^{\prime \prime} 54^{\circ} 44^{\prime} 23.7^{\prime \prime} \mathrm{W}$ & 0,20 & 177 \\
Melipona seminigra & Jacarandá do Pará & $\mathrm{S} 2^{\circ} 25^{\prime} 12.2^{\prime \prime} 54^{\circ} 44^{\prime} 21.9^{\prime \prime} \mathrm{W}$ & 2,33 & 181 \\
Trigona pallens & Copaibarana & $\mathrm{S} 2^{\circ} 25^{\prime} 8.9^{\prime \prime} \mathrm{S} 54^{\circ} 44^{\prime} 25.1^{\prime \prime} \mathrm{W}$ & 1,02 & 151 \\
& & Bosque Mekdece & & \\
Melipona interrupta & Ingá & $\mathrm{S} 2^{\circ} 25^{\prime} 11.9^{\prime \prime} 54^{\circ} 44^{\prime} 20.9^{\prime \prime} \mathrm{W}$ & 4,47 & 123 \\
Tetragona clavipes & Ingá & $\mathrm{S} 2^{\circ} 25^{\prime} 11.9^{\prime \prime} 54^{\circ} 44^{\prime} 20.9^{\prime \prime} \mathrm{W}$ & 3,65 & 123 \\
Scaptotrigona sp. & Ingá & $\mathrm{S} 2^{\circ} 25^{\prime} 11.9^{\prime \prime} 54^{\circ} 44^{\prime} 20.9^{\prime \prime} \mathrm{W}$ & 1,55 & 123 \\
Trigona pallens & solo & $\mathrm{S} 2^{\circ} 25^{\prime} 12.8^{\prime \prime} 54^{\circ} 44^{\prime} 20^{\prime \prime} \mathrm{W}$ & solo & solo \\
\hline
\end{tabular}

Nos dois locais de estudo observou-se uma variação da altura dos ninhos em relação ao solo (Tabela 1). M. interrupta foi a espécie que teve a maior variação: de 0,95 a 6,00m, o único ninho de $M$. seminigra estava a uma altura de 2,33m. Trigona pallens teve a menor variação: de 0,20 a 1,02 m e Tetragona clavipes teve uma variação de 3,65 a 6,00 m. O único ninho de Scaptotrigona sp estava a uma altura de 1,55 m. Em geral, a estrutura interna dos ninhos de meliponíneos em troncos de árvore fica próxima à entrada dos ninhos, assim a altura da entrada é uma referência para a altura dos ninhos como um todo (WILLE e MICHENER,1973). O estudo mostrou a construção preferencial do ninho em posições mais baixas por Trigona pallens e em posições mais altas por Melipona interrupta. 
Também foi verificado, nos dois locais de estudo, que as espécies de abelhas nidificaram em substratos arbóreos com Circunferência à altura do peito (CAP) maior que $120 \mathrm{~cm}$ (Figuras 7 e 8). No Campus Tapajós, M. seminigra nidificou em árvore com CAP equivalente a 181 cm, T.clavipes e M. interrupta nidificaram em árvores com CAP entre 120 e 143 cm (valor dado pela média), T. pallens nidificou em árvores com CAP entre 150 e 177 cm (Figura 8). A correlação de Pearson entre o CAP das espécies arbóreas e o número de ninhos resultou em 7\%, ou seja, representa que houve baixa correlação entre os parâmetros observados, entretanto estatisticamente, a baixa densidade de ninhos encontrados não permite verificar de fato se existe ou não correlação no local de estudo. No Bosque Mekdece, das quatro espécies encontradas, apenas três: Scapitotrigona sp, T. clavipes e M. interrupta, nidificaram na mesma árvore, que apresentou CAP de 123 cm (Figura 9). Neste local não foi possível calcular a correlação.

Neste estudo não houve relação entre o CAP das árvores e a altura dos ninhos, mas houve uma grande variação em relação à altura dos ninhos, variando entre as faixas de 0,20 e $6 \mathrm{~m}$. Em Correia et al. (2016), a correlação linear obtida foi negativa $(r=-0,095 p=0,652)$, não mostrando relação significativa entre CAP e altura dos ninhos, contudo, evidenciou diferentes alturas de nidificação que varia de 0 a $4 \mathrm{~m}$, preferencialmente entre as faixas de CAP de 60 a $200 \mathrm{~cm}$.

Figura 8. Distribuição dos ninhos em função do CAP no Campus Tapajós, Santarém, Pará, Brasil.

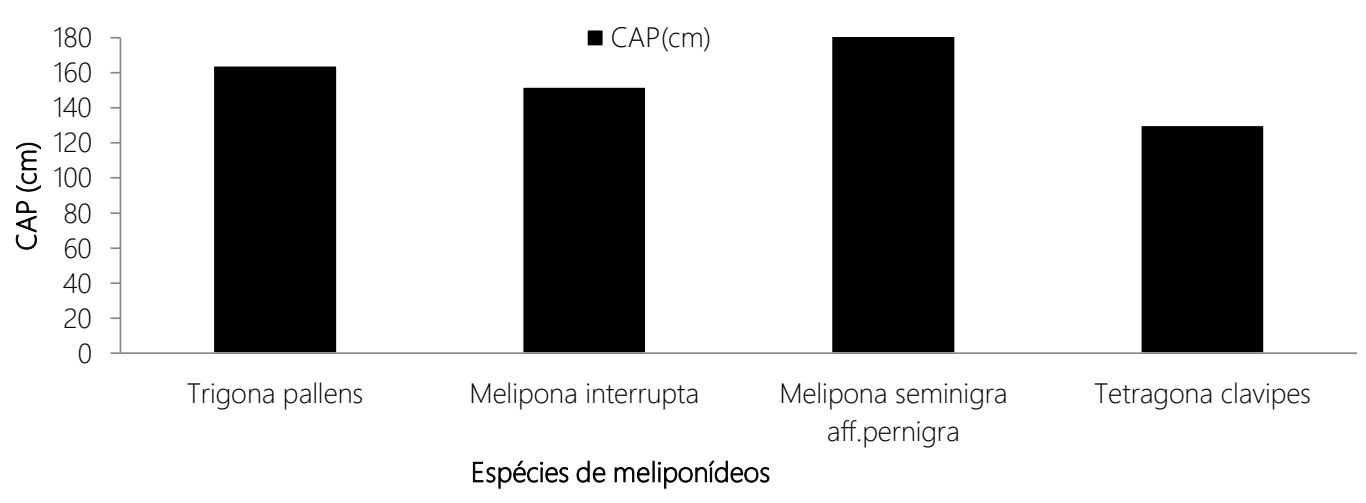


Figura 9. Distribuição dos ninhos em função do CAP no Bosque Mekdece, Santarém, Pará, Brasil.

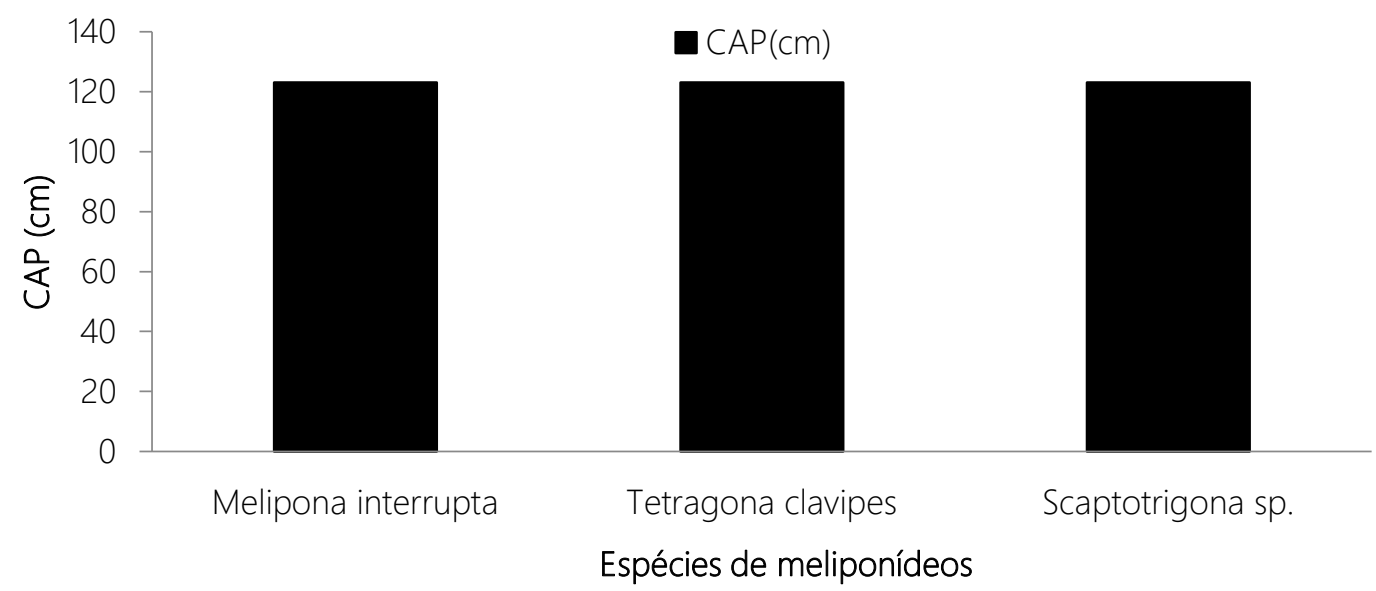

Os índices de diversidade ShannonWiener $\left(H^{\prime}\right)$ foram respectivamente $H^{\prime}=$ 1,56 no Campus Tapajós e $H^{\prime}=1,04$ no Bosque Mekdece, já os índices de Equitabilidade de Pielou (J) foram J=0,97 no Campus Tapajós e J=0,95 no Bosque Mekdece. Constatou-se pelo Índice de diversidade Shannon Wiener $\left(H^{\prime}\right)$ que a diversidade foi baixa nos dois locais (VILHENA, 2009) e as espécies estão distribuídas de forma homogênea, ou seja, não existe dominância entre elas. Tonhasca et al. (2002) encontrou resultado semelhante, porém em florestas de Mata Atlântica.

A densidade dos ninhos encontrada foi de 1 ninho por hectare no Campus
Tapajós e 3 ninhos por hectare no Bosque Mekdece, entretanto não foi encontrado relação entre a diversidade de abelhas e o tamanho do fragmento florestal em Correia et al. (2016) e Antonini et al. (2013), em seus estudos grandes fragmentos não contêm mais espécies por unidade de área do que os menores, porém, em pequenos fragmentos as abelhas são as mais comuns e possuem comportamentos generalistas assim como não foi constatado relação entre a riqueza de espécies com o tamanho dos fragmentos das florestas urbanas (ANTONINI et al., 2013). 
Tabela 2. Estudos sobre a densidade de ninhos de abelhas sem ferrão realizados no Brasil.

\begin{tabular}{lcccc}
\hline Autores & Estado & Área (ha) & $N^{\circ}$ de ninhos & Densidade (ha) \\
\hline Este estudo & Pará & 12 - Campus & 8 & 1 \\
& & $1,4-$ Bosque & 4 & 3 \\
Correia et al.(2016) & Acre & 137 & 25 & 0,18 \\
Souza (2014) & São Paulo & 4,2 & 14 & 3,33 \\
Silva et al.(2013) & Bahia & 5,4 & 35 & 6,5 \\
Serra et al.(2009) & Maranhão & 185,5 & 73 & 2,6 \\
Siqueira et al.(2007) & Minas Gerais & 50,4 & 21 & 2,4 \\
Souza et al.(2005 b) & Bahia & 57 & 94 & 1,6 \\
Antonini e Martins (2003) & Minas Gerais & 171,4 & 48 & 0,28 \\
Teixeira (2001) & Bahia & 5 & 147 & 29,4 \\
& & & & \\
\hline
\end{tabular}

\section{CONCLUSÃO}

Constatou-se que a maioria dos ninhos estavam localizados em árvores. Desta forma o número de árvores existentes no Campus Tapajós e no Bosque Mekdece podem favorecer a predominância de abelhas sem ferrão, principalmente as espécies do gênero Melipona. Por apresentar regiões ocas, algumas árvores aumentam a oportunidade de locais para nidificação, oferecendo condições propícias ao abrigo das Meliponini.

- presente trabalho demonstrou a importância da preservação de áreas urbanas para a conservação da biodiversidade de abelhas sem ferrão.

\section{AGRADECIMENTOS}

À Universidade Federal do Oeste do Pará (UFOPA) pelo apoio financeiro.

\section{REFERÊNCIAS}

ANTONINI, Y.; MARTINS, R. The value of a tree species (Caryocar brasiliense) for a stingless bee Melipona quadrifasciata quadrifasciata. Journal of Insect Conservation, v.7, p.167-174. 2003.

ANTONINI, Y.; MARTINS, R. P.; AGUIAR, L. M.; LOYOLA, R. D. Richness, composition and trophic niche of stingless bee assemblage in urban forest remnants. Urban Ecosystem, v.16, p.527-541, 2013.

AQUINO, F. G.; WALTER, B. M. T.; RIBEIRO, J. F. Woody community dynamics in two fragments of "cerrado" stricto sensu over a seven-year period (1995-2002), MA, Brazil. Revista Brasileira de Botânica, v. 30, p.113-121, 2007. 
BATISTA, M.A.; RAMALHO, M.; SOARES, A.E.E. Nesting sites and abundance of Meliponini (Hymenoptera: Apidae) in heterogeneous habitats of the Atlantic Rain Forest, Bahia. Revista Lundiana, v.4, p. 19-23, 2003.

BROWN, J. C.; ALBRECHT, C. The effect of tropical deforestation on stingless bees of the genus Melipona (Insecta: Hymenoptera: Apidae: Meliponini) in central Rondônia, Brazil. Journal of Biogeography, v.28, p.623-624, 2001.

CAMARGO, J. M. F. Ninhos e biologia de algumas espécies de meliponídeos (Hymenoptera: Apidae) da região de Porto Velho, território de Rondônia, Brasil.

Revista de Biologia Tropical, v.2, p. 207239, 1970.

CAMARGO, J. M. F.; ROUBIK, D. W. Systematics and bionomics of the apoid obligate necrophages: the Trigona hypogea group (Hymenoptera: Apidae, Meliponinae). Biological Journal of the Linnean Society, v.44, p.13-39, 1991.

CAMARGO, J. M. F.; PEDRO, S. R. M. Notas sobre a bionomia de Trichotrigona extranea (Hymenoptera, Apidae, Meliponini). Revista Brasileira de Entomologia, v. 51, p.72- 81, 2007.

CAMARGO J. M. F.; PEDRO, S. R.M. Revisão das espécies de Melipona do grupo fuliginosa (Hymenoptera, Apoidea, Apidae, Meliponini). Revista Brasileira de Entomologia, v. 52, p. 411-427, 2008.

CORREIA, F. C. da S.; PERUQUETTI, R. C.; FERREIRA, M. G.; CARVALHO, Y. K. Abundância, Distribuição Espacial de
Ninhos de Abelhas Sem Ferrão (Apidae: Meliponini) e Espécies Vegetais Utilizadas para Nidificação em um Fragmento de Floresta Secundária em Rio Branco, Acre. Revista EntomoBrasilis, v.9, p.163-168, 2016.

ELTZ, T.; BRÜHL, C. A.; IMIYABIR, Z.; LINSENMAIR, K.E. Nesting and nest trees of stingless bees (Apidae: Meliponini) in lowland dipterocarp forests in Sabah, Malaysia, with implications for forest management. Forest Ecology and Management, v. 172, p. 301-313, 2003.

FRAZÃO, R. F. Biodiversidade Amazônica: A fauna de Abelhas nativas (Hymenoptera: Apidae: Meliponina) da Região Sul da Floresta Nacional do Amapá, Relação com flores e Potenciais de Uso, Amapá, 2009. 61 f. Dissertação (Mestrado Integrado em Desenvolvimento Regional) Universidade Federal do Amapá, Amapá, 2009.

GONÇALVES, S.J.M.; RÊGO, M.; ARAÚJO, A. Abelhas sociais (Hymenoptera: Apidae) e seus recursos florais em uma região de mata secundária, Alcântara, Maranhão. Acta Amazônica, v.26, p. 55-68, 1996.

HUBBELL, S. P.; JOHNSON, L. K. Competition and nest spacing in a tropical stingless bee community. Ecology, v. 58, p. 949-963, 1977.

INMET- Instituto Nacional de Meteorologia. Disponível em INMETInstituto Nacional de Meteorologia< http://www.inmet.gov.br/>. Acesso em: 09 julho de 2017. 
KERR, W. E.; FILHO, A. B. Meliponíneos. Revista Biotecnologia Ciência e Desenvolvimento, v.8, p.22-23, 1999.

LINSLEY, E. G. The ecology of solitary bees. Hilgardia, v. 27, p. 543-599, 1958.

MARTINS, C. F.; CORTOPASSI, M. L.; KOEDAM, D.; IMPERATRIZ FONSECA, V. L. Espécies arbóreas utilizadas para nidificação por abelhas sem ferrão na caatinga (Seridó, PB; João Câmara, RN). Revista Biota Neotropical, v.4, p. 1-8, 2004.

MARTINS, A. C.; AGUIAR, A. J. C.; SANTOS I.A. dos. Interaction between oil-collecting bees and seven species of Plantaginaceae. Revista Flora, v.208, p. 401-411, 2013.

NOGUEIRA-NETO, P. Vida e criação de abelhas indígenas sem ferrão. São Paulo (SP): Editora Nogueirapis, 1997, vol. 1. 445 p.

OLIVEIRA, M. L.; MORATO, E. F.; GARCIA, M. $\vee$. B. Diversidade de espécies e densidade de ninhos de abelhas sociais sem ferrão (Hymenoptera: Apidae, Meliponinae) em floresta de terra firme na Amazônia Central. Revista Brasileira de Zoologia, v. 12, p. 13-24, 1995.

OLIVEIRA, M. A.; AIDAR, D. S. Efeito da alimentação artificial no crescimento de colônias de Melipona seminigra merrillae (Hymenoptera, Apidae, Meliponinae). Revista Mensagem Doce, v.89, p. 16-26, 2006.

OLIVEIRA, F. F.; RICHERS, B. T. T.; SILVA, J. R.; FARIAS, R. C.; MATOS, T. A. L. Guia ilustrado das abelhas "sem-ferrão" das Reservas Amanã e Mamirauá, Amazonas,
Brasil (Hymenoptera, Apidae, Meliponini). Instituto de Desenvolvimento Sustentável Mamirauá, Tefé, Amazonas, 2013. 270 p.

RÊGO, M. M. C.; BRITO, C. Abelhas sociais (Apidae: Meliponini) em um ecossistema de cerrado (Chapadinha - MA, BR): distribuição dos ninhos. In: ENCONTRO SOBRE ABELHAS, 3. Ribeirão Preto. Anais... Ribeirão Preto, SP, v.3, p.238-247, 1996.

RÊGO, M.M. C.; ALBUQUERQUE, P.M.C.; VENTURIERI, G. Menos Locais para ninhos. Revista Ciência Hoje, v. 247, p.50-51, 2008.

ROUBIK, D.W. Ecology and natural history of tropical bees. Cambridge University Press, Cambridge, 1989. 514 p.

ROUBIK, D. W. Stingless bee nesting biology. Apidologie, v.37, p.124-143, 2006.

SERRA, B. D. V.; DRUMMOND, M. S.; LACERDA, L. M.; AKATSU, I. P. Abundância, distribuição espacial de ninhos de abelhas Meliponina (Hymenoptera, Apidae, Apini) e espécies vegetais utilizadas para nidificação em áreas de cerrado do Maranhão. Revista Série Zoológica, v. 99, p. 12-17, 2009.

SILVA, M.D.; RAMALHO, M.; MONTEIRO, $D$. Diversity and habitat use by stingless bees (Apidae) in the Brazilian Atlantic Forest. Apidologie, v. 44, p. 699-707, 2013.

SILVEIRA, F. A; MELO, G. A. R.; ALMEIDA, E. A. B. Abelhas brasileiras: sistemática e identificação. Belo Horizonte, Ministério do Meio Ambiente, v. 1. 253 p, 2002.

SIQUEIRA, E.L., MARTINES, R.B.; NOGUEIRA-FERREIRA, F.H. Ninhos de 
Abelhas sem Ferrão (Hymenoptera, Meliponina) em uma região do rio Araguari, Araguari-MG. Bioscience Journal, v. 23, p. 134-140, 2007.

SLAA, E. J. Population dynamics of a stingless bee community in the seasonal dry lowlands of Costa Rica. Insectes Sociaux, v.53, p.70-79, 2006.

SOUZA, A.K.P.; HERNÁNDEZ, M.I.M.; MARTINS, C.F. Riqueza, abundância e diversidade de Euglossina (Hymenoptera, Apidae) em três áreas da Reserva Biológica Guaribas, Paraíba, Brasil. Revista Brasileira de Zoologia, v. 22, p. 320-325, 2005a.

SOUZA, S.G.X.; TEIXEIRA, A.F.R.; NEVES, E.L.; MELO, A.M.C. As abelhas sem ferrão (Apidae: Meliponina) residentes no Campus Federação/Ondina da Universidade Federal da Bahia, Salvador, Bahia, Brasil. Candombá - Revista Virtual, v.1, p. 57-69, 2005b.

SOUZA, C .S. Riqueza, abundância relativa e densidade de ninhos de meliponíneos (Apidae, Meliponini) em duas áreas de estágios sucessionais distintos de vegetação do Parque Estadual das Fontes do Ipiranga, São Paulo (23³8'S; 46³8'36W), São Paulo, 2014. $83 \mathrm{f}$. Dissertação (Mestrado em Ciências Ecologia) - Departamento de ecologia do Instituto de Biociências da Universidade de São Paulo, São Paulo, 2014.

TEIXEIRA, A. F. R. Abelhas indígenas (Hymenoptera: Meliponini) residentes em uma área de caatinga na margem esquerda do Rio São Francisco, Salvador, 2001.42 f. Monografia (Bacharelado em
Ciências Biológicas) - Instituto de Biologia, Universidade Federal da Bahia, Salvador, 2001.

TEIXEIRA, A. F. R. Ecologia das abelhas eussociais do gênero Frieseomelitta von Ihering, 1912 (Apidae; Meliponina), Salvador, BA. 2003. 108 f. Dissertação (Mestrado em Ecologia e Biomonitoramento) - Instituto de Biologia da Universidade Federal da Bahia, Salvador, 2003.

TONHASCA, A.J.; BLACKMER, J.L.; ALBUQUERQUE, G.S. Abundance and Diversity of Euglossine Bees in the Fragmented Landscape of the Brazilian Atlantic Forest. Biotropica, v.34, p. 416422, 2002.

VERGARA, C.B.; VILLA, A.L.; NATES, G.P. Nidificación de meliponinos (Hymenoptera: Apidae) de la Región Central de Colômbia. Revista Biología Tropical. v. 34, p.181-184, 1986.

VILHENA, P. S. Fauna de Abelhas do Torrão do Matapí, Amapá, Brasil, Amapá, 2009.56 f. Trabalho de Conclusão de Curso de Graduação em Ciências Biológicas-Universidade Federal do Amapá-UNIFAP, Amapá, 2009.

WILLE, A.; MICHENER, C. D. The nest architecture of stingless bees with special reference to those of Costa Rica (Hymenoptera: Apidae). Revista de Biologia Tropical, v.9, p. 278, 1973. 